\title{
ARTICLE Reversal of peripheral nerve injury-induced neuropathic pain and cognitive dysfunction via genetic and tomivosertib targeting of MNK
}

\author{
Stephanie Shiers ${ }^{1}$, Juliet Mwirigi ${ }^{1}$, Grishma Pradhan ${ }^{1}$, Moeno Kume ${ }^{1}$, Bryan Black ${ }^{2}$, Paulino Barragan-Iglesias (D) ${ }^{1}$, Jamie K. Moy ${ }^{1}$, \\ Gregory Dussor ${ }^{1}$, Joseph J. Pancrazio ${ }^{2}$, Sven Kroener ${ }^{1}$ and Theodore J. Price ${ }^{1}$
}

\begin{abstract}
Neuropathic pain caused by nerve injury presents with severe spontaneous pain and a variety of comorbidities, including deficits in higher executive functions. None of these clinical problems are adequately treated with current analgesics. Targeting of the mitogen-activated protein kinase-interacting kinase (MNK1/2) and its phosphorylation target, the mRNA cap binding protein elF4E, attenuates many types of nociceptive plasticity induced by inflammatory mediators and chemotherapeutic drugs but inhibiting this pathway does not alter nerve injury-induced mechanical allodynia. We used genetic manipulations and pharmacology to inhibit MNK-eIF4E activity in animals with spared nerve injury, a model of peripheral nerve injury (PNI)-induced neuropathic pain. We assessed the presence of spontaneous pain using conditioned place preference. We also tested performance in a medial prefrontal cortex (mPFC)-dependent rule-shifting task. WT neuropathic animals showed signs of spontaneous pain and were significantly impaired in the rule-shifting task while genetic and pharmacological inhibition of the MNK-elF4E signaling axis protected against and reversed spontaneous pain and PNI-mediated cognitive impairment. Additionally, pharmacological and genetic inhibition of MNK-elF4E signaling completely blocked and reversed maladaptive shortening in the length of axon initial segments (AIS) in the mPFC of PNI mice. Surprisingly, these striking positive outcomes on neuropathic pain occurred in the absence of any effect on mechanical allodynia, a standard test for neuropathic pain efficacy. Our results illustrate new testing paradigms for determining preclinical neuropathic pain efficacy and point to the MNK inhibitor tomivosertib (eFT508) as an important drug candidate for neuropathic pain treatment.
\end{abstract}

Neuropsychopharmacology (2020) 45:524-533; https://doi.org/10.1038/s41386-019-0537-y

\section{INTRODUCTION}

Neuropathic pain is characterized by spontaneous pain originating from peripheral nerve injury (PNI) and it is accompanied by disruptions in executive functions that depend on an intact medial prefrontal cortex (mPFC) [1-7]. Importantly, existing therapeutics are often ineffective for spontaneous pain, moreover, effective pain relief with existing analgesics usually does not improve painrelated cognitive dysfunction $[1,4,5,8,9]$. As such, there is a need for new therapeutics that can treat neuropathic pain and its cognitive comorbidities. The goal of this study was to identify a therapeutic target that can alleviate PNI-induced spontaneous pain, as well as its associated negative influence on cognition.

$\mathrm{PNI}$, chemotherapy, and inflammatory mediators cause augmented signaling in translation control pathways in peripheral nociceptors that are responsible for detecting potentially damaging stimuli $[6,10-14]$. The consequence of activation of these pathways is increased excitability and spontaneous activity in nociceptors, both of which are key drivers of neuropathic pain in animal models and in patients [15-17]. The initiation of translation is regulated at multiple levels by integrating eukaryotic initiation factor (eIF) complexes that facilitate the translation of specific subsets of mRNAs $[18,19]$. In the nervous system two key translational signaling pathways have been shown to regulate this step of protein synthesis: the mammalian target of rapamycin (mTOR) and mitogen-activated protein kinase (MAPK) pathways. In the MAPK signaling pathway, MAPK-interacting serine/threonine kinase 1 and 2 (MNK1/2) phosphorylates eIF4E which leads to the selective translation of mRNAs involved in neuronal plasticity. Among these mRNAs are known mediators of neuropathic pain such as Bdnf and Mmp9 [20-23]. Genetic or pharmacological targeting of elF4E activity leads to a reduction in mechanical and thermal hypersensitivity and affective pain behaviors induced by inflammatory mediators or chemotherapeutic drugs [11, 14]. However, the impact of this pathway on PNI-evoked pain is less clear because genetic reduction of elF4E phosphorylation has little effect on mechanical allodynia at later time points after PNI ( $>2$ weeks) but does reduce cold allodynia [11].

We recently showed that mice with $\mathrm{PNI}$ are significantly impaired in a mPFC-dependent rule-shifting task which assesses cognitive flexibility [6]. This cognitive phenotype is associated with axon initial segment (AIS) shortening in neurons of layer $5 / 6$ of the infralimbic cortex, linking behavioral changes to a specific neuronal pathology. Here we measured PNI-induced changes in rule-shifting and used conditioned place preference (CPP) to

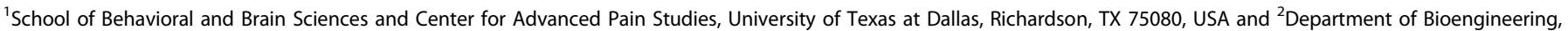
University of Texas at Dallas, Richardson, TX 75080, USA

Correspondence: Theodore J. Price (Theodore.price@utdallas.edu)
}

Received: 6 May 2019 Revised: 18 September 2019 Accepted: 25 September 2019

Published online: 7 October 2019 
assess spontaneous pain in mice with a mutation at the MNK1/2 phosphorylation site of elF4E (4EKI), a global MNK1 knock-out (MNK1 KO), as well as mice treated with the MNK1/2 inhibitor tomivosertib (eFT508). Our findings demonstrate that genetic and pharmacological targeting of the elF4E signaling pathway reverses pain-related cognitive impairment, spontaneous pain, and rescues AIS shortening in the MPFC in PNI-induced neuropathic pain. Surprisingly, all of these effects are observed without an effect on mechanical allodynia, raising important questions about the utility of this commonly used measure of neuropathic pain in mice.

\section{METHODS}

Animals

Animals were housed on a 12-h light/dark cycle and had food and water available ad libitum. For experiments involving rule-shifting, animals were individually housed for the purposes of food restriction. All procedures were performed during the light cycle. All experiments were approved by the Institutional Animal Care and Use Committee at the University of Texas at Dallas and conducted in accordance with the National Institutes of Health and International Association for the Study of Pain guidelines. 4EKI and MNK1 KO mice were a gift of the Sonenberg laboratory at McGill University $[20,24]$ and bred at the University of Texas at Dallas (UTD) to generate experimental animals. Genotype was confirmed at weaning using DNA from ear clips. Experimental C57BL6/J wild-type (WT) animals were obtained from an internally maintained $\mathrm{C} 57 \mathrm{BL} / 6 \mathrm{~J}$ colony at the University of Texas at Dallas. Twelve-week-old mice were used for all histology and rule-shifting experiments due to food restriction requirements [25]. All western blot, marble burying, and CPP behavioral experiments were conducted in 4-8-week-old mice with SNI being conducted at 4-5 weeks old, and all behavioral end points being observed at 7-8 weeks old. Male mice were used in all rule-shifting and immunohistochemistry experiments, except for a single experiment comparing male and female 4EKI mice, due to our previously published work showing a sex difference in rule-shifting performance in which males are significantly more impaired than females [6]. As we have never observed a sex difference in pain behaviors associated with pharmacological or genetic manipulations of the MNK-elF4E system [11, 12, 14, 23], we chose to use male and/or female mice in our experiments. The sex of the animal used in each experiment is indicated on the graphs. Experimenters performing behavioral experiments were blinded to genotypes and drug treatments.

\section{Drugs}

Tomivosertib (also known as eFT508) was provided by eFFECTOR under a Material Transfer agreement with UTD. Per eFFECTOR instructions, tomivosertib was dissolved in 10\% 1-Methyl-2pyrrolidinone, anhydrous (NMP; Sigma Cat\# 328634), 90\% Propylene Glycol (PG; Fisher Cat\# P355-1) with $\sim 1$ eq. of $\mathrm{HCl}$. Tomivosertib was given orally to WT mice at $10 \mathrm{mg} / \mathrm{kg}$ daily for $7 \mathrm{~d}$ for all rule-shifting, marble burying, von Frey, and histology experiments with the last injection occurring $1 \mathrm{~h}$ before behavioral testing. For the CPP experiment, tomivosertib was given intraperitoneally (i.p.) at $10 \mathrm{mg} / \mathrm{kg}$, but at a lower volume to avoid sedation from the drug vehicle ( $50 \mu \mathrm{L}$ per injection). We confirmed this volume of tomivosertib, or vehicle did not anesthetize the animals by conducting rotarod and open field on a mixed-sex cohort of naïve, vehicle, and tomivosertib-treated mice (Fig S2). Intrathecal (i.t.) injections of clonidine (Sigma) were administered at $10 \mu \mathrm{g} / 5 \mu \mathrm{L}$, dissolved in $0.9 \%$ sterile saline.

\section{Conditioned place preference}

A previously published CPP protocol from the Walters laboratory protocol was used [15]. The CPP box consisted of one black and one white chamber connected by a striped chamber. CPP was conducted in a dark room with a single low lumens light source (Maia 69E120-WH). The middle chamber of the CPP box was brightly illuminated to discourage mice from spending time in the middle chamber. The black and white end chambers had a different floor texture and odor (scented chapsticks applied to the ceiling). On day 1 of testing (preconditioning), each mouse was permitted to explore the middle and black (but not white) chambers for $30 \mathrm{~min}$. Conditioning occurred on days 2-4, except for a single experiment using tomivosertib which had only one conditioning day. On conditioning days, the mice were given either vehicle (tomivosertib experiments; i.p.) or nothing (clonidine experiments) and then $1 \mathrm{~h}$ later they were confined to the black chamber for $30 \mathrm{~min}$. Three hours later, the mice were given an injection of tomivosertib or vehicle (i.p., $10 \mathrm{mg} / \mathrm{kg}$ at $4 \mathrm{mg} / \mathrm{mL}$ ) or clonidine (i.t., $10 \mu \mathrm{g} / 5 \mu \mathrm{L}$ ), and $1 \mathrm{~h}$ later they were confined to the white chamber for $30 \mathrm{~min}$. On the last day of CPP, each mouse was placed in the middle chamber with unrestricted access to all chambers and video-recorded for $10 \mathrm{~min}$. Data are presented as the percent change in time spent in the drug-paired chamber compared to the control (vehicle or sham for each genotype or surgery). This was calculated by dividing each animal's time spent in the white box by the control average, multiplying by 100 and then subtracting 100 .

\section{Surgery}

Spared nerve injury (SNI) was performed by the ligation and cutting of the tibial and peroneal branches of the left sciatic nerve trifurcation, leaving the sural branch intact as previously described [26]. Sham surgeries were performed the same way but without cutting the nerve. Mice were allowed to recover for 2 weeks following surgery.

\section{Mechanical withdrawal threshold}

Tactile sensitivity was measured by probing the left outer surface of the left hindpaw with a series of calibrated von Frey filaments. Mechanical withdrawal thresholds were assessed in all animals before (baseline) and after ( 2 weeks) surgery to confirm neuropathy. Withdrawal thresholds were calculated using the up-down method [27].

\section{Attentional rule-shifting}

Rule-shifting was performed as previously described [6]. Ruleshifting took place on day 21 post-SNI for all groups. For ruleshifting experiments with tomivosertib, the drug was delivered orally $(10 \mathrm{mg} / \mathrm{kg}$ ) every day for 7 days with rule-shifting occurring $1 \mathrm{~h}$ after the final injection.

Marble burying task

The marble burying task was used to assess obsessive compulsive disorder (OCD)-like behaviors as previously described [28]. Nineteen marbles were placed on top of $5 \mathrm{~cm}$ of standard bedding in a clean cage. A mouse was placed into the cage for $30 \mathrm{~min}$ and then the number of buried marbles was counted.

\section{Immunohistochemistry}

All mice were perfused at 3 weeks post-surgery (the day after ruleshifting). Mice were deeply anesthetized with a mixture of ketamine $(80 \mathrm{mg} / \mathrm{kg})$ and xylazine $(12 \mathrm{mg} / \mathrm{kg}$; i.p.) and then transcardially perfused with $1 \mathrm{X}$ phosphate buffered saline (PBS; $4{ }^{\circ} \mathrm{C}, \mathrm{pH} 7.4$ ) followed by $10 \%$ formalin in $0.1 \mathrm{M}$ phosphate buffer $\left(\mathrm{PB} ; 4{ }^{\circ} \mathrm{C}, \mathrm{pH} 7.4 ; 25 \mathrm{~mL}\right.$ at a flow rate of $5 \mathrm{~mL} / \mathrm{min}$ ) using a perfusion pump. Brains were dissected and transferred to a $30 \%$ sucrose in $0.1 \mathrm{M} \mathrm{PB}$. Once the brains sunk in sucrose, they were frozen in OCT over dry ice. Coronal sections $(30 \mu \mathrm{m})$ were cut using a cryostat and a hole was placed in the left hemisphere to demarcate the left (ipsilateral) hemisphere. Free floating sections were then washed in $0.1 \mathrm{M} \mathrm{PB}$, and then incubated in blocking solution (10\% Normal Goat Serum, 0.3\% Triton-X 100 in 0.1 M PB) 
for $1 \mathrm{~h}$ at room temperature with gentle rocking/agitation. Sections were incubated in primary antibody (mouse-anti-AnkyrinG; UC Davis/NIH NeuroMab Facility, clone N106/36 RRID: AB_10673030; $2 \mu \mathrm{g} / \mathrm{ml}$ working concentration) diluted in blocking solution for $3 \mathrm{~h}$ at room temperature. Sections were washed five times in $0.1 \mathrm{M} \mathrm{PB}$, and then incubated in secondary antibody (goat-anti-mouse 488 lgG2a, Thermo Fisher Scientific, A21131;1:2000) diluted in blocking solution containing DAPI (1:5000) for $1 \mathrm{~h}$ at room temperature. Sections were washed five times in 0.1 M PB, mounted onto glass slides, cover-slipped using Prolong Gold Antifade (Thermo Fisher Scientific, P36930) and sealed with nail polish once cured.

\section{Rotarod}

Motor function was assessed using a rotarod (IITC Life Science rotarod series 8 ) with ramp speed ( $20 \mathrm{rpm}$ acceleration) at a starting speed of $4 \mathrm{pm}$ for a maximum of $120 \mathrm{~s}$. The time until each animal fell was recorded for each trial. Naïve mice were trained for 1 trial, and then 2 baseline trials were conducted and scores for both trials were averaged. Tomivosertib $(10 \mathrm{mg} / \mathrm{kg}$ i.p $\sim 50 \mu \mathrm{L}$ ) or vehicle was then injected, and $1 \mathrm{~h}$ later all mice were tested twice more, and their scores were averaged.

\section{Open field}

Naïve, tomivosertib (10 mg/kg i.p. $\sim 50 \mu \mathrm{L}$ ) or vehicle-treated mice were tested in an open field $(60 \mathrm{~cm} \times 60 \mathrm{~cm}$ white box) $1 \mathrm{~h}$ after injection. Movement in the maze was video recorded for $10 \mathrm{~min}$ via ceiling-mounted cameras and tracked using a custom script in MATLAB-2015b. The script outputs total distance traveled and path tracking.

\section{Image analysis}

Axon initial segments (AIS - Ankyrin-G antibody). A minimum of three z-stacked images of infralimbic cortex (IL) were acquired at $\times 40$ magnification for each animal using an Olympus confocal microscope (FV1200). Images were converted to tiff format with randomized file names (for blinding). Images were brightened and contrasted with the same settings across all groups. For each image, 30 segments were analyzed in layers $2 / 3$ and layers $5 / 6$ of the ipsilateral and contralateral IL in CellSens (Olympus) using the polyline measure tool. Length measurements were averaged (three images per animal). $\times 100$ images are also shown in the figures.

Western blotting. Naïve WT mice were decapitated following anesthesia $1 \mathrm{~h}$ after tomivosertib (p.o. or i.p.) or vehicle injection. The mPFC and lumbar dorsal root ganglia (DRGs) were rapidly microdissected under a dissecting microscope and then flash frozen on dry ice. The mPFC was similarly collected from a separate group of WT male and female sham and SNI mice at 21 days post injury (the same time point as all rule-shifting experiments). Frozen tissues were homogenized with a pestle in lysis buffer ( $50 \mathrm{mM}$ Tris, pH 7.4, $150 \mathrm{mM} \mathrm{NaCl}, 1$ mM EDTA, pH 8.0, and $1 \%$ Triton $\mathrm{X}-100)$ containing protease and phosphatase inhibitors (Sigma-Aldrich), and then sonicated briefly. Samples were centrifuged at $14,000 \mathrm{rpm}$ for $15 \mathrm{~min}$ at $4{ }^{\circ} \mathrm{C}$. In all, $10 \mu \mathrm{g}$ of protein was loaded into each well and separated by a $10-12 \%$ SDS-PAGE gel. Proteins were transferred to a $0.45 \mu \mathrm{m}$ PVDF membrane (Millipore) at $30 \mathrm{~V}$ overnight at $4{ }^{\circ} \mathrm{C}$. The next day, membranes were blocked with $5 \%$ non-fat dry milk (NFDM) in $1 \mathrm{X}$ Tris buffer solution containing Tween 20 (TTBS) for $1 \mathrm{~h}$. Membranes were washed in $1 \mathrm{X}$ TTBS three times for 5 min each, then incubated with primary antibody overnight at $4{ }^{\circ} \mathrm{C}$. The primary antibodies used were: phospho-elF4E (abcam ab76256; 1:1000), GAPDH (Cell Signaling Technology 2118 S; 1:10,000), total elF4E (Cell Signaling Technology 9742 S; 1:1000). The following day, membranes were washed three times in 1X TTBS for 5 min each, then incubated with the corresponding secondary antibody at room temperature for $1 \mathrm{~h}$. Membranes were then washed with $1 \times$ TTBS 5 times for $5 \mathrm{~min}$ each. Signals were detected using Immobilon Western Chemiluminescent HRP substrate (Millipore). Bands were visualized using a Bio-Rad ChemiDoc Touch. Membranes were stripped using Restore Western Blot Stripping buffer (Thermo Fisher Scientific) and reprobed with another antibody. Analysis was performed using Image Lab (Bio-Rad). Data are presented as p-elF4E/total elF4E normalized to control.

Extracellular electrophysiology. Substrate-integrated microelectrode array (MEA) recordings were performed using PDL and laminin pre-treated 12-well MEA plates (Axion Biosystems, USA) and an Axion Maestro recording system. DRG sensory neurons were dissected, dissociated, and seeded as previously described [11]. Tomivosertib experiments were carried out 15 days after DRG culture seeding. Cultures were maintained at $37{ }^{\circ} \mathrm{C}, 5 \% \mathrm{CO}_{2}$, and $\geq 90 \%$ humidity throughout the recordings. Briefly, $30 \mathrm{~min}$ baseline recordings were carried out following a $30 \mathrm{~min}$ acclimation period. Following the baseline recording period, cultures were treated with either interleukin-6 (IL-6; $100 \mathrm{ng} / \mathrm{ml}$ final concentration; $n=$ 11 wells) only, IL-6 plus tomivosertib ( $25 \mathrm{nM}$ final concentration; $n=11$ wells), or tomivosertib only ( $n=7$ wells). Recordings were then collected continuously for $3 \mathrm{~h}$. IL- 6 and tomivosertib were added at 1:1000 as high-concentration boluses. Online and offline MEA data analysis was performed using Axion's AxIS, NeuralMetrics Tools, and NeuroExplorer (NeuroExplorer, USA). Physiologically relevant extracellular action potentials were defined as previously described [29].

Data analysis and statistics

Graphs and statistical analyses were generated using GraphPad Prism version 7.01, except for the MEA experiment which was assessed using Axion's AxIS, NeuralMetrics Tools, and NeuroExplorer. Statistical differences between groups were assessed using one- and two-way ANOVAs followed by Bonferroni corrected multiple $t$-tests for analysis with more than three groups or unpaired $t$-tests for tests with only two groups as indicated in the text and figure captions. All statistics including $F$ and exact p-values are shown in supplementary tables (Tables S3, S4, and S5). All data are represented as mean \pm SEM with $p<0.05$ considered significant. The sample size and sex of all subjects are indicated in the graphs and/or figure captions.

\section{RESULTS}

Genetic inhibition of elF4E phosphorylation rescues pain-related cognitive impairment in an attentional rule-shifting task

We used a rule-shifting task (Fig. 1a) to assess neuropathic painrelated impairments in cognitive flexibility in WT and 4EKI mice, a genetically modified mouse strain in which the MNK phosphorylation site on elF4E is mutated to render it phosphorylation null. On response training day, groups showed no differences in acquisition of the response training strategy (Fig. 1b). The next day, the animals were given SNI or sham surgeries and they were then retested on the original response strategy 20 days postsurgery. Male 4EKI and WT animals showed no deficits on retest day, indicating that neither surgery nor elF4E phosphorylation disrupts retention of the initial response strategy (Fig. 1b). On day 21 post-surgery, all groups were required to switch strategies (rule-shifting) to receive food reward. Male WT SNI mice were significantly impaired on rule-shifting day when compared to WT sham, 4EKI SNI, and 4EKI sham mice (Fig. 1b). Male SNI WT mice made significantly more never-reinforced (NRE) and regressive (REG) type errors, but not perseverative errors (PER), compared to WT sham controls and 4EKI sham and SNI mice, suggesting there was an issue with maintenance of the new strategy (Fig. 1c). We have previously shown a large sex difference in rule-shifting performance with males being significantly more impaired than 
A

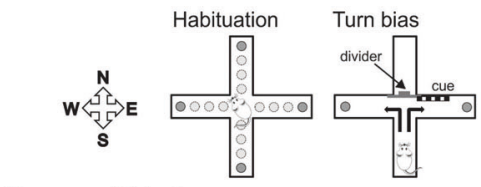

Response / Retest

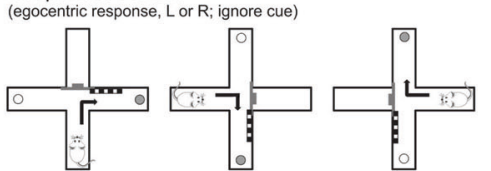

Rule-shifting (turn towards visual cue)

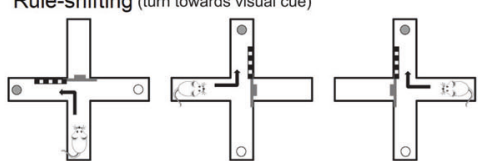

C

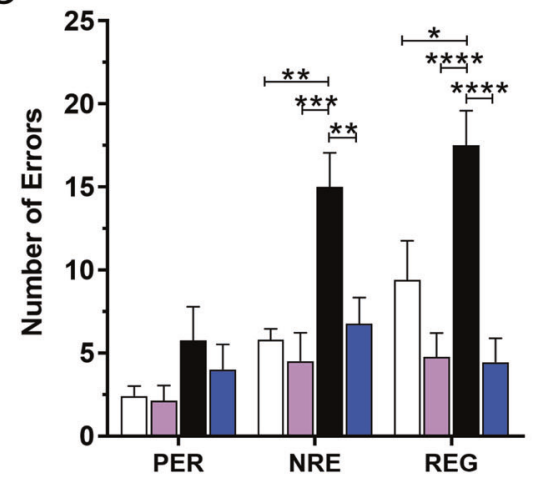

B

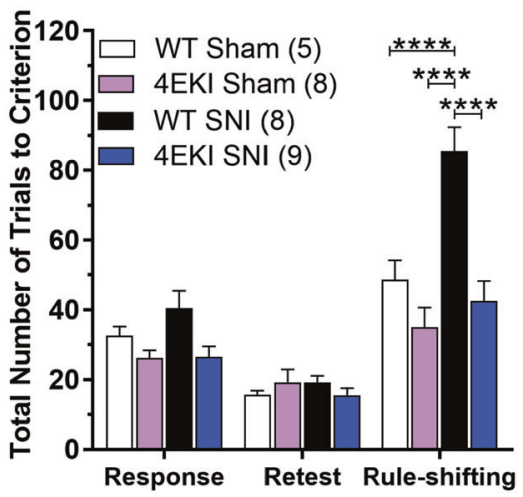

D

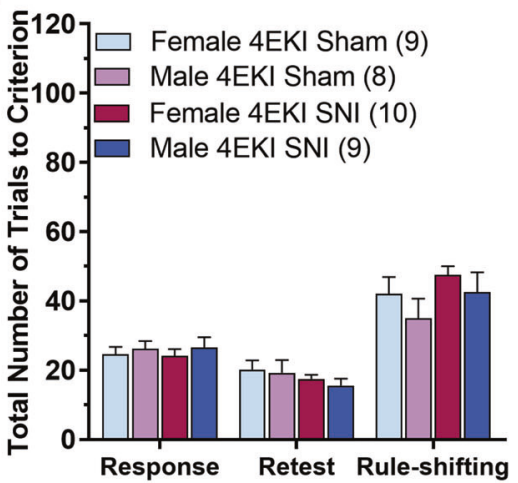

Fig. 1 Genetic inhibition of elF4E phosphorylation rescues pain-related cognitive impairment in an attentional rule-shifting task. a Illustration of the rule-shifting protocol. Mice were habituated to the maze over 3 days. On day 4, mice were assessed for preference to turn right or left over 7 trials (Turn bias). The following day, mice were trained to turn against their turn bias in order to retrieve food reward and to ignore the pseudorandomly placed visual cue (Response). Approximately 3 weeks after SNI, the animals were assessed for retention of the task (Retest). The next day, mice were trained to shift strategies so that they now had to turn towards the arm containing the visual cue to obtain food reward (Rule-shifting). b Male 4EKI SNI mice showed no impairment on retest day, indicating that disruption of eiF4E phosphorylation has no effect on retention of response training. Male 4EKI SNI mice displayed no impairment on rule-shifting day. c Male 4EKI SNI mice made significantly fewer never-reinforced errors (NRE) and regressive errors (REG), but not perseverative errors (PER), compared to WT SNI animals. d Male and female 4EKI mice displayed no differences on rule-shifting day. Two-way ANOVA with Bonferroni multiple comparisons ${ }^{* * *} p<$ $0.0001,{ }^{* * *} p<0.001,{ }^{* *} p<0.01,{ }^{*} p<0.05$

females in the attentional rule-shifting task [6], so we focused most of our rule-shifting experiments on male mice. However, when we tested female 4EKI mice, female SNI mice showed no impairment on response, retest, nor rule-shifting day when compared to female sham or male sham or SNI mice (Fig. 1d).

Disruption of elF4E phosphorylation prevents PNI-induced spontaneous pain

Female WT and 4EKI mice with sham or SNI surgeries were assessed for ongoing pain in a CPP apparatus in which the conditioning drug was clonidine, an a2 adrenergic receptor agonist [30]. Following conditioning, WT SNI animals spent significantly more time ( $30 \%$ more time) in the clonidinepaired chamber compared to WT sham controls, suggesting that the chamber was rewarding due to analgesia. 4EKI SNI mice showed no significant difference in time spent in the drug-paired chamber compared to 4EKI sham controls (Fig. 2a), indicating that the loss of elF4E phosphorylation protects against the development of spontaneous pain following PNI (Fig. 2a). WT and 4EKI SNI animals were mechanically hypersensitive when withdrawal thresholds were assessed 14 days post-surgery (Fig. 2b). Importantly, clonidine significantly attenuated mechanical hypersensitivity when assessed $2 \mathrm{~h}$ after injection on each CPP conditioning day (Fig. 2b), demonstrating that clonidine is pharmacologically active in both genotypes. These experiments indicate that although 4EKI mice show mechanical allodynia after PNI, they fail to display signs of spontaneous pain.

Pharmacological and genetic inhibition of MNK reverses PNIinduced impairments in rule-shifting Experiments in 4EKI mice implicate elF4E phosphorylation in PNIinduced spontaneous pain and rule-shifting deficits but they do not determine whether these effects can be reversed via inhibition of the MAPK signaling pathway once PNI-neuropathic pain is wellestablished. We hypothesized that pharmacological inhibition of MNK1/2, the only two known kinases responsible for phosphorylation of elF4E, would fully reverse rule-shifting deficits. Before surgery and drug treatment naïve WT male animals were trained on the response strategy. There was a significant difference in baseline performance in this cohort of mice; however, these differences disappeared once animals successfully learned the original discrimination, and accordingly no behavioral differences were observed on Retest Day (Fig. 3a). Two weeks following surgery, WT SNI and sham animals were given a daily treatment of either vehicle or tomivosertib (10 mg/kg p.o.) for 7 days. Retest took place on day 6 of injections, and all groups showed no differences in retention of response training (Fig. 3a). Rule-shifting took place $1 \mathrm{~h}$ after the final injection on day 7. SNI animals treated with tomivosertib showed no cognitive impairment on rule-shifting day when compared to SNI + vehicle animals (Fig. 3a). 

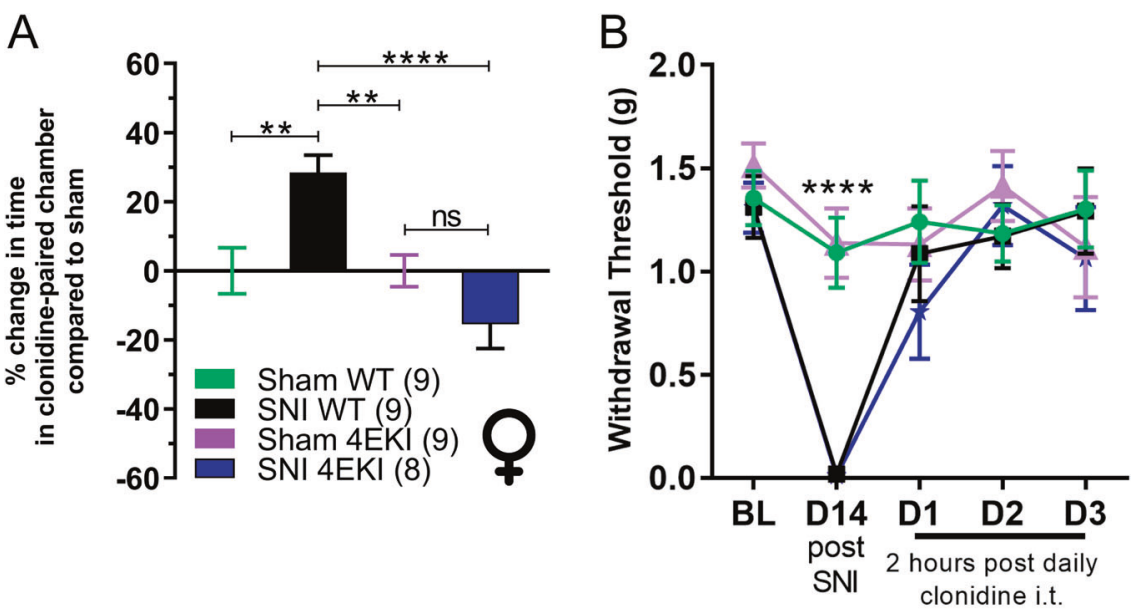

(10ug)

Fig. 2 Disruption of elF4E phosphorylation in 4EKI mice prevents PNI-induced spontaneous pain. a WT and 4EKI SNI and sham mice underwent a CPP paradigm in which clonidine $(10 \mu \mathrm{g}$ i.t.) was paired with the white chamber over 3 conditioning days. WT SNI mice spent significantly more time in the analgesic-paired chamber compared to WT sham mice. However, 4EKI SNI animals and 4EKI sham mice showed no difference in time in spent in the analgesic-paired chamber. These data suggest that 4EKI mice have reduced spontaneous pain. b Clonidine $(10 \mu \mathrm{g}$ i.t.) reversed mechanical withdrawal thresholds when assessed $2 \mathrm{~h}$ after injection on each CPP conditioning day. One or two-way ANOVA with Bonferroni multiple comparisons ${ }^{* * *} p<0.0001,{ }^{* *} p<0.01$

Tomivosertib-treated SNI animals made significantly fewer NRE and REG type errors compared to the SNI + vehicle group (Fig. 3b). Despite the profound effect on PNI-induced rule-shifting deficits, tomivosertib-treatment had no effect on mechanical withdrawal thresholds when assessed 1 or $3 \mathrm{~h}$ after treatment on days 1 and 5 of the 7-day treatment schedule (Fig. 3c).

Tomivosertib acts via both MNK1 and 2. Our previous experiments in chemotherapy-induced neuropathic pain suggest that MNK1 is a key isoform driving pain induced by chemotherapy [14]. To test whether MNK1 plays a similar dominant role in PNIinduced neuropathic pain, we used a full-body knockout strain for MNK1. We tested these mice in the rule-shifting task. Naive WT and MNK1 KOs mice showed no differences in performance on response training day, indicating knockout of the MNK1 gene does not impair acquisition of the initial response strategy (Fig. 3d). However, when tested 20 days after surgery (retest day), the MNK1 KO animals showed significantly impaired retention of response training, suggesting that MNK1 may be important for long-term memory (Fig. 3d). However, MNK1 KO mice with SNI showed no deficit in rule-shifting performance (Fig. 3d) and made significantly fewer NRE and REG errors when compared to WT SNI animals (Fig. 3e). Similar to previous reports in neuropathic 4EKI mice [11], MNK1 KO SNI animals showed a slight attenuation in tactile allodynia 4 days after surgery compared to WT SNI animals but showed no differences to WT SNI animals on days 7, 11, 14, and 17 post SNI (Fig. 3f).

While the number of trials to criterion during rule-shifting did not differ from WT sham mice, MNK1 KO SNI animals took significantly longer to complete the rule-shift, with each trial taking 1.5-2 min on average to complete (Fig S1A). WT mice took less than $1 \mathrm{~min}$, on average. These MNK1 KO animals appeared distracted in the maze and were grooming, staring at walls, or repetitively jumping to escape the maze, leading to the prolonged rule-shifting time. We did not observe the same type of behaviors during rule-shifting in either the 4EKI mice (not shown), nor in the tomivosertib-treated animals (Fig S1B). We used a marble burying task to test if these behaviors were reflective of obsessive compulsive disorder (OCD)-like traits. The tomivosertib-treated and vehicle controls showed no differences in marble burying compared to the WT naïve group (Fig S1C); however, the 4EKI and MNK1 KO mice buried significantly more marbles than WT naïve mice, suggesting that disruption of elF4e signaling throughout development causes OCD-like symptoms (Fig S1C). Additionally, we observed another OCD-like behavior in 4EKI and MNK1 KO mice (but not WT animals) in which these animals display stereotypic jumping behaviors, especially during their dark cycle (Supplemental video file 1).

Pharmacological targeting of MNK1/2 reverses PNI-induced spontaneous pain

The pharmacokinetics of tomivosertib have been described in detail [31]; however, in order to understand if tomivosertib can reduce spontaneous pain in PNI mice, we examined the onset of tomivosertib effects after p.o. or i.p. dosing. As elevated elF4E phosphorylation has been previously observed in DRG neurons and their axons in chronic pain conditions [10, 14], we investigated how tomivosertib affected elF4E phosphorylation in lumbar (L3-L5) DRG, sciatic nerve, and mPFC. Western blot analysis of $\mathrm{mPFC}, \mathrm{DRG}$, and sciatic nerve from naïve mice revealed a rapid and profound decrease in elF4E phosphorylation $1 \mathrm{~h}$ after tomivosertib treatment via both administration routes (Fig. $4 a$ ).

Having determined that tomivosertib acts rapidly to inhibit elF4E phosphorylation after i.p. dosing, we sought to determine if the drug could induce CPP in animals with SNI. To do this we used a single conditioning day of tomivosertib or vehicle (i.p) pairings with the white chamber. SNI animals that received tomivosertib in the white chamber spent $\sim 40 \%$ more time in that chamber on test day compared to SNI animals that only received vehicle. This $40 \%$ increase in time was significantly different than the change in time in the sham-tomivosertib-treated animals (Fig. 4b). This result supports the conclusion that MNK1/2 inhibition with tomivosertib can reduce spontaneous pain caused by PNI.

Although we used oral dosing of tomivosertib for the ruleshifting experiment, we used i.p. injections in our CPP experiment to avoid a potential stress-aversion to the conditioning chamber brought about by the gavage. The concentration of the drug was raised in order to deliver a smaller volume of vehicle and drug as the vehicle given at high volumes intraperitoneally can anesthetize the animal (note: oral injections do not have this effect). The mice appeared active in their cages $1 \mathrm{~h}$ after drug-administration at the lower volume and we confirmed this observation by open field (Fig S2A) and by rotarod (Fig S2C). No significant differences were seen between naïve, vehicle nor tomivosertib-treated mice in the rotarod test. Total distance traveled and movement speed 

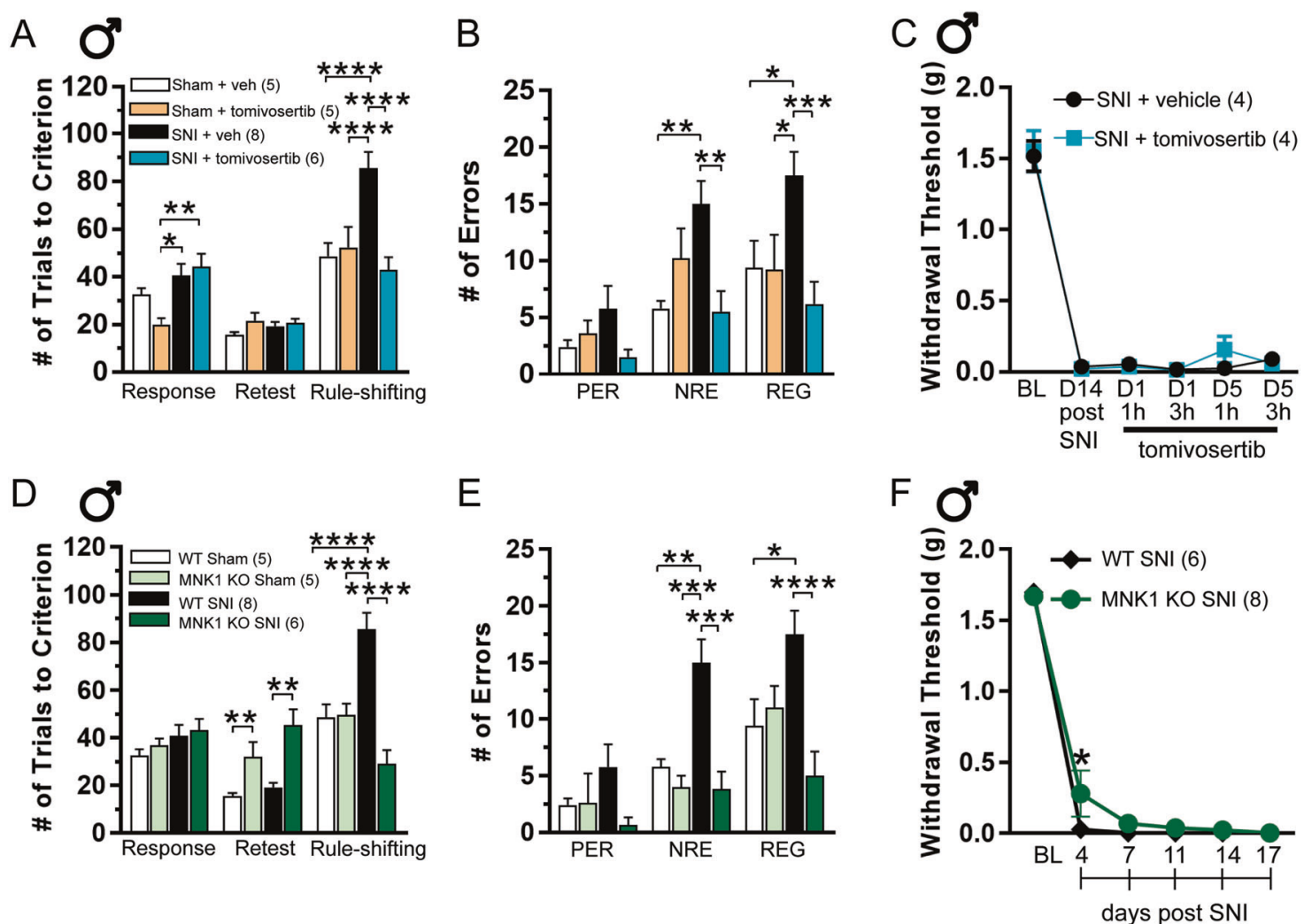

Fig. 3 Pharmacological and genetic inhibition of MNK reverses PNI-induced impairment in attentional rule-shifting. a WT SNI mice were treated with the MNK1/2 inhibitor, tomivosertib (10 mg/kg, p.o.), or vehicle once daily for 7 days. Rule-shifting took place $1 \mathrm{~h}$ after the final injection. Tomivosertib-treated mice showed no impairment on rule-shifting day. b WT SNI vehicle-treated animals made significantly more never-reinforced errors (NRE) and regressive errors (REG), but not perseverative errors (PER) compared to all other groups. c Tomivosertibtreatment had no effect on mechanical withdrawal thresholds when assessed 1 or $3 \mathrm{~h}$ after treatment on days 1 and 5 of the 7-day treatment schedule. d MNK1 KOs displayed a significant impairment in retention of response training on retest day, suggesting that disruption of MNK1 signaling causes issues with long-term memory. However, the MNK1 KO SNI mice showed no impairment on rule-shifting day. e MNK1 KO SNI animals also made significantly fewer NRE and REG when compared to WT SNI mice. f MNK1 KO SNI animals showed a slight attenuation in tactile allodynia on day 4 post surgery compared to WT SNI animals but showed no differences to WT SNI animals on days 7 , 11 , 14 , and 17 post SNI. Two-way ANOVA with Bonferroni multiple comparisons ${ }^{* * *} p<0.0001,{ }^{* * *} p<0.001,{ }^{* *} p<0.01,{ }^{*} p<0.05$

was decreased in the vehicle and tomivosertib-treated mice compared to naïve controls in the open field; however, they were still active as exemplified in their exploration maps (Fig S2B).

Given the CPP effect, we assessed whether tomivosertib could reduce nociceptor firing and elucidate a mechanism for the reduction in spontaneous pain. We used microelectrode array recordings of cultured mouse DRG neurons exposed to interleukin-6 (IL-6), a cytokine that evokes pain via an action that is dependent on nociceptor expression of its receptor gp130 and that depends on elF4E phosphorylation. Tomivosertib significantly reduced spontaneous firing induced by IL- 6 treatment (Fig. 4c).

Finally, we assessed if elF4E phosphorylation is changed in the mPFC after SNI in male or female mice. Our previous work has demonstrated that elF4E phosphorylation is profoundly upregulated in the DRG of chemoneuropathic mice [14] and in the sciatic nerve of SNI mice and rats over a similar time course to experiments performed here [10]. In contrast to the peripheral changes in elF4E phosphorylation brought about by PNI, we did not observe a difference in elF4E phosphorylation in the mPFC 3 weeks after injury (Fig. 4d).

Inhibition of elF4E signaling reverses PNI-induced changes in AIS length in the infralimbic cortex

Structural changes in AIS length are linked to changes in neuronal excitability and homeostatic plasticity. Increased or decreased presynaptic input will shorten or lengthen, respectively, the AIS and shift the action potential initiation site to reduce or augment neuronal excitability [32-34]. We have previously shown that SNI leads to a reduction in AIS length in layer $5 / 6$ of the infralimbic cortex ipsilateral and contralateral to the site of SNI. This AIS shrinkage correlates to deficits in rule-shifting performance caused by PNI [6]. We assessed if this structural plasticity was also present in $4 \mathrm{EKI}, \mathrm{MNK} 1 \mathrm{KO}$, and tomivosertib-treated animals. Tomivosertib-treated animals were given p.o. treatments $(10 \mathrm{mg} /$ $\mathrm{kg}$ ) for 7 days starting on day 14 after SNI. We observed axon initial segment shrinkage in layer $5 / 6$ of the ipsilateral and contralateral infralimbic cortices (Fig. 5a) in WT SNI (Fig. 5b) male mice, but not male 4EKI (Fig. 5c), male MNK1 KO (Fig. 5d), nor tomivosertib-treated male SNI mice (Fig. 5e). This finding correlates with behavioral performance after $\mathrm{SNI}$ in these treatment groups and strongly supports the idea that spontaneous pain may drive rule-shifting deficits and MPFC pathology after PNI.

\section{DISCUSSION}

Cognitive dysfunction is a debilitating comorbidity of chronic pain which diminishes quality of life in patients. Commonly prescribed analgesics often have no effect or even worsen pain-related cognitive impairment, indicating that there is a disconnect between the management of pain and its comorbidities $[1,4,5,35,36]$. Human brain neuroimaging [37-40] and preclinical studies $[6,41-46]$ have revealed that chronic pain causes 


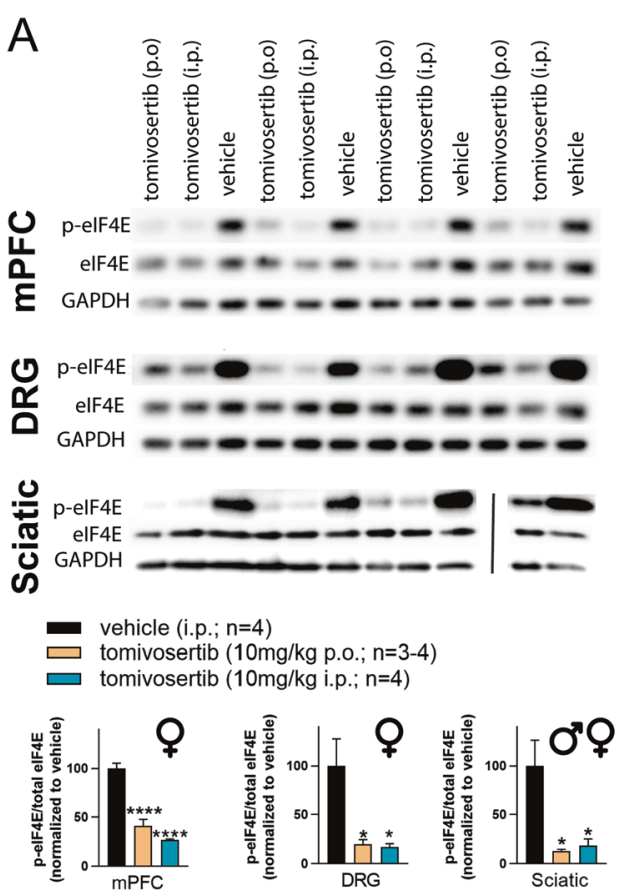

B
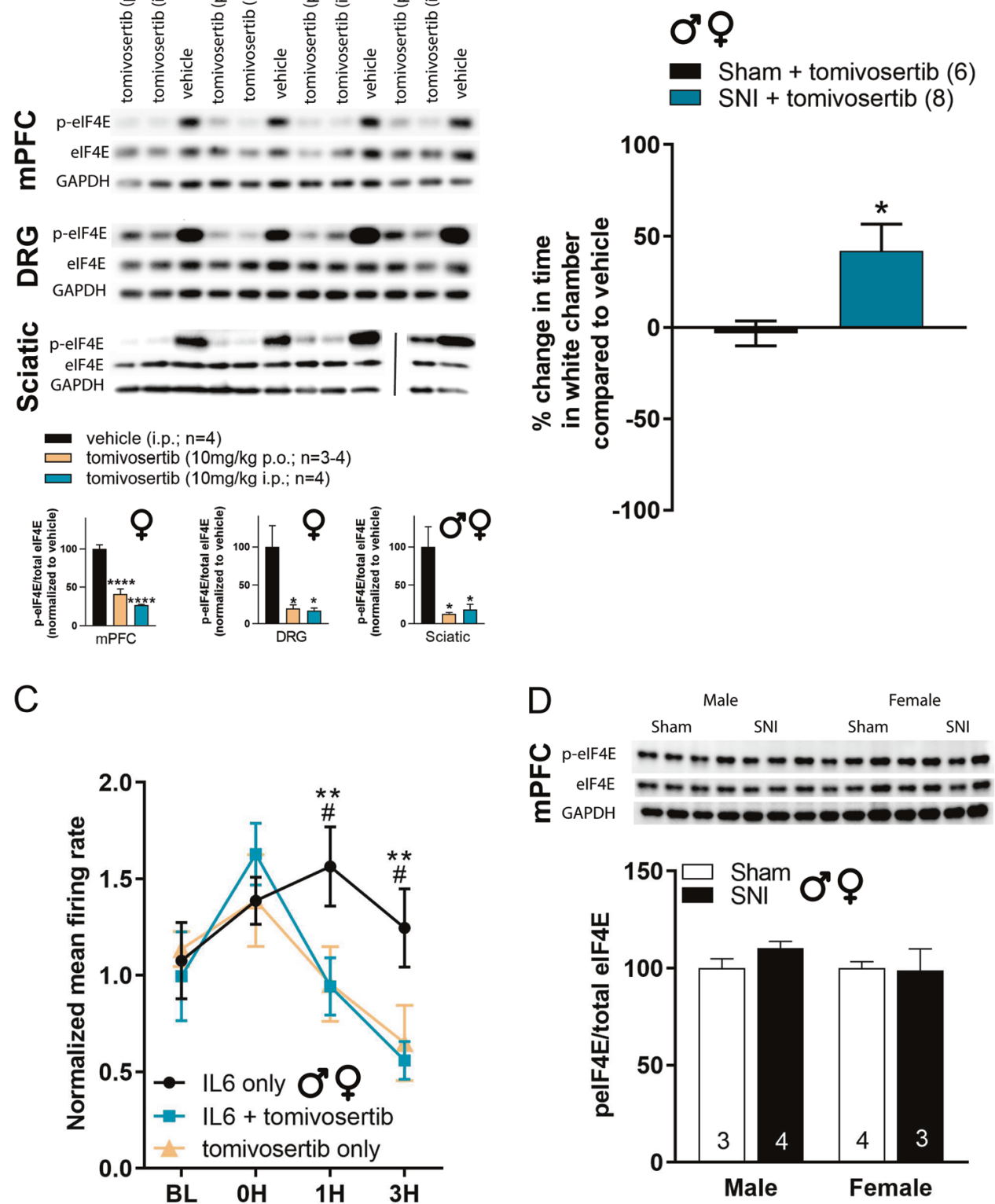

Fig. 4 Pharmacological inhibition of MNK1/2 with tomivosertib reverses PNI-induced spontaneous pain. a Western blot analysis of elF4e phosphorylation was conducted on mPFC, DRG, and sciatic nerve collected from animals $1 \mathrm{~h}$ after administration of tomivosertib (10 mg/kg; i. $\mathrm{p}$ or p.o.) or vehicle. Phosphorylation of elF4E was significantly decreased in mPFC, DRG, and sciatic nerve after tomivosertib treatment. b WT SNI animals were tested in a CPP paradigm using tomivosertib $(10 \mathrm{mg} / \mathrm{kg}$ i.p.) as the conditioning-drug. SNI animals treated with tomivosertib spent $\sim 40 \%$ more time in the white chamber compared to SNI animals that received only vehicle. This $40 \%$ increase in time was significantly different from the change in time between tomivosertib and vehicle conditioned sham mice, suggesting that tomivosertib diminishes spontaneous pain caused by PNI. c Microelectrode array recordings of cultured mouse DRG neurons exposed to interleukin-6 (IL-6) showed enhanced spontaneous firing which was attenuated by co-treatment with tomivosertib ( $25 \mathrm{nM})$. MEA dishes that were only treated with tomivosertib showed a reduction in firing compared to the IL- 6 only group. For the tomivosertib group, there was no significant difference in firing rate between any of the treatment time points and the baseline recording $(p>0.05)$. **IL6 only vs IL6 + tomivosertib; \#IL6 only vs tomivosertib only. d Western blot analysis revealed that elF4E phosphorylation was unchanged in mPFC after SNI in male and female mice. One-way ANOVA with Bonferroni multiple comparisons (blot and MEA data), unpaired $t$-test (CPP) ${ }^{* * * *} p<0.0001,{ }^{* *} p<0.01,{ }^{*} p<0.05$

functional and morphological reorganization of prefrontal cortex subregions which may contribute to pain-induced cognitive dysfunction. However, the underlying mechanisms behind these changes are still unknown, making it difficult to generate new therapeutics that can simultaneously target pain and comorbidities of the disease. Our findings show that targeting MNK1-eIF4E signaling inhibits and reverses rule-shifting deficits associated with $\mathrm{PNI}$, and it reduces spontaneous pain, which is the most important clinical complaint of neuropathic pain patients. We conclude that the specific MNK1/2 inhibitor tomivosertib has great potential to impact neuropathic pain.

An important question is whether the effects we have observed here are mediated by a central or peripheral site of action. We hypothesize that the site of action is likely peripheral. Previous studies in multiple neuropathic pain models in both rats and mice have demonstrated that elF4E phosphorylation is increased in DRG neurons and their axons over the course of neuropathic pain $[10,14]$. Moreover, electrophysiological experiments using 

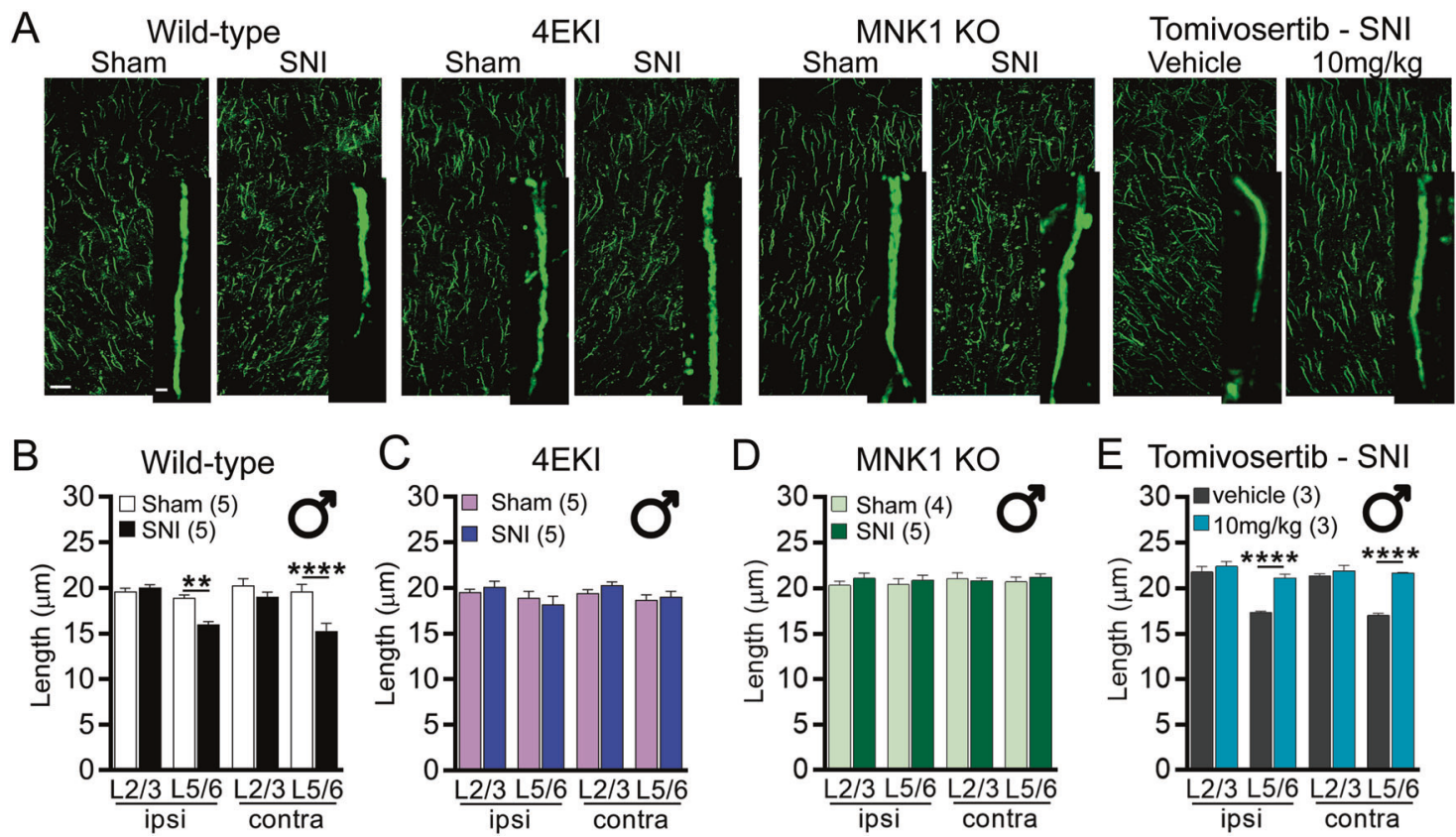

Fig. 5 Inhibition of elF4E signaling reverses PNI-induced changes in AIS length in the infralimbic cortex. a Representative images of AIS staining in the infralimbic cortex of WT, 4EKI, MNK1 KO, and tomivosertib-treated (10 mg/kg p.o. daily for 7 days) animals. b AIS length was significantly decreased in L5/L6 of the IL in WT SNI male. SNI did not alter AIS length in c 4EKI SNI mice nor d MNK1 KO SNI animals. SNIinduced changes in AIS length were reversed by e tomivosertib treatment. Image scale bar: $20 \mu \mathrm{m}$ and $500 \mathrm{~nm}$, respectively. Two-way ANOVA with Bonferroni multiple comparisons ${ }^{* * *} p<0.0001,{ }^{* *} p<0.01$

tomivosertib and other MNK inhibitors, as well as genetic experiments in 4EKI mice, have shown that many cytokines and growth factors associated with neuropathic pain fail to sensitize nociceptors when MNK signaling is blocked [11]. This also includes reversal of spontaneous activity in nociceptors from mice treated with chemotherapy [14]. Insofar as spontaneous activity in nociceptors is likely the key driver of spontaneous pain in rodent models and in patients suffering from neuropathic pain, MNK inhibition might be particularly suited to address this most salient feature of neuropathic pain. In support of this conclusion, we found that tomivosertib-treatment resolved ectopic activity after IL-6 treatment in cultured DRG neurons and induced CPP in neuropathic mice.

Genetic and pharmacological targeting of elF4E also reversed deficits in rule-switching performance. Our interpretation of these findings is that disruption of MNK-elF4E signaling in the DRG is leading to a resolution of ectopic activity in nociceptors, and of ascending nociceptive signaling to the brain, resulting in improved cognitive function. Indeed, ascending nociceptive projections terminate in several key brain regions that project to the mPFC and are implicated in cognitive dysfunction in pain. The most compelling evidence supports a central and basolateral amygdala (CeA-BLA) projection in which nociceptive information arising from the spino-parabrachio-amygdala pain pathway [47] leads to enhanced activity in CeA [48] and BLA neurons after injury $[43,49]$. BLA hyperactivity in models of arthritic pain leads to enhanced glutamatergic signaling to the PrL resulting in augmented feedforward inhibition, PrL deactivation, and cognitive impairment [43]. It is likely that resolution of nociceptor ectopic activity via MNK-elF4E inhibition corrects this enhanced nociceptive drive, leading to restoration of cognitive function in the ruleshifting task.

Importantly, we did not see signs of increased MNK-elF4E signaling in the mPFC of SNI mice, suggesting that the MPFC is unlikely to be the site of action of genetic or pharmacological interventions. However, tomivosertib clearly crosses the blood-brain-barrier as evidenced by our western blot analyses of elF4E phosphorylation after drug-treatment. Therefore, it is possible that a decrease in eIF4E phosphorylation from baseline levels could be responsible for restoration of cognitive function after SNI; however, we do not have evidence to support this conclusion. Additionally, it is possible that elF4E signaling could be disrupted in other regions that project to the MPFC. To distinguish between central and peripheral contributions, nociceptor-specific manipulation of MNK1 and development of a peripherallyrestricted MNK1/2 inhibitor will be a priority for future investigations.

While we favor a peripheral hypothesis, we cannot rule out an effect of eIF4E phosphorylation in the CNS. elF4E dysregulation has been identified in a variety of neuropsychiatric disorders including autism, fragile $\times$ syndrome, schizophrenia, and depression/anxiety [50]. MNK-elF4E signaling appears to regulate serotonin neurotransmission in the brain, and deficits in elF4E phosphorylation can lead to depressive phenotypes [22]. Those effects can be recapitulated with pharmacological treatments in adult animals [51]. While genetic manipulation of MNK-elF4E caused OCD-like behaviors in our experiments, pharmacological inhibition of MNK using tomivosertib, even over a week of treatment, did not have an effect. This suggests that the OCD-like phenotype we observed in both genotypes is developmentally regulated and is unlikely to arise with pharmacological treatment in adults.

It is notable that we observed strong effects on spontaneous pain and attentional rule shifting without any clear effect on mechanical allodynia. This is likely explained by a difference in the type of afferent fibers that are required for mechanical allodynia in different models. Mechanical allodynia, as measured with punctate stimulation with traditional von Frey filaments, is absent in inflammatory mouse models when all nociceptors are ablated, whereas SNI-induced mechanical allodynia persists in the absence of these fibers [52]. This indicates that $A \beta$ fibers are sufficient to mediate mechanical allodynia following SNI. On the other hand, 
cold allodynia requires the TRPM8-population of nociceptors [53]. In MNK KO and 4EKI mice many forms of inflammatory mechanical allodynia and cold hypersensitivity after SNI are strongly attenuated [11]. This indicates that nociceptors are most strongly affected by inhibition of MNK-elF4E signaling. Insofar as nociceptor spontaneous activity is a key driver of spontaneous neuropathic pain [17], and inhibition of MNK-elF4E signaling also reduces this form of intrinsic plasticity in these cells [14], this effect likely explains why MNK inhibition reduces spontaneous pain. This effect on spontaneous pain occurs within $1.5 \mathrm{~h}$ in vivo as shown by the CPP experiment time course. Our previous work demonstrates that MNK inhibition leads to resolution of spontaneous activity in nociceptors taken from mice with neuropathic pain within $20 \mathrm{~min}$ [14]. It is interesting that such a strong effect on spontaneous pain cannot influence mechanical allodynia in neuropathic pain, but this may reflect a lack of effect of MNK-eIF4E signaling on spinal disinhibition which is a key driver of $A \beta$-fiber driven allodynia after PNI [54].

Change in AIS length is a homeostatic mechanism for neurons to maintain neuronal excitability by regulating the expression of voltage-gated channels $[55,56]$. We observed AIS shrinkage in bilateral layer $5 / 6 \mathrm{IL}$ neurons which correlated to poor performance in the rule-shifting task. While we did not use cell-specific markers to identify which cortical neurons we were measuring, we are confident that the majority, if not all, of the AISs measured were from pyramidal neurons. Pyramidal cells have a very unique, organized and defined cytoarchitecture in which their axons travel distally from the cell body while interneurons have axons that can stem in any direction. We only measured axons that showed a pyramidal neuron-like AIS label. AIS shrinkage is a sign of enhanced presynaptic input. Previous studies found that IL neurons show no changes in functional activity 1 week after SNI $[46,57]$ but this may change at later time points where AIS shrinkage is clearly occurring in the IL.

We speculate that this AIS loss may partially underlie frontal cortex gray matter loss seen in chronic pain patients. Others have observed changes in dendritic morphology $[41,45,57]$ in rodent mPFC after SNI, suggesting that neuronal structural reorganization may underlie cortical thinning. It is important to note that there are only two methods known to reverse cortical thinning in patients: cognitive behavioral therapy [58] and effective pain management [59-61]. Cortical gray matter restoration has been observed in patients with hip osteoarthritic pain whose pain dissipated after undergoing total hip replacement surgery $[59,60]$ and in chronic low back pain patients after spinal surgery or facet joint injections [61]. Increases in cortical thickness in chronic low back pain patients, particularly in the left dorsolateral prefrontal cortex, the rodent equivalent of the mPFC, correlated to a reduction in pain and physical disability and improved cognitive performance in an attentional-demanding task [61]. Similarly, in our neuropathic mice, effective, long-term pain management via both tomivosertib and metformin [6], an AMPK activator and upstream regulator of elF4E activity, attenuated both cognitive deficits and AIS shrinkage in the IL.

We conclude that MNK-eIF4E signaling plays a crucial role in the generation of spontaneous pain and rule-shifting deficits that develop as a consequence of PNI. Surprisingly, these important features of neuropathic pain can be mitigated with genetic or pharmacological approaches without having any effect on mechanical allodynia. Because mechanical allodynia is the most commonly utilized test for neuropathic pain in preclinical models, our results demonstrate that this approach can overlook potential therapeutic approaches that target the most salient complaints of neuropathic pain: spontaneous pain and cognitive comorbidities. Our results make a strong case for further exploration of tomivosertib, which is already in human clinical trials for cancer, as a novel neuropathic pain treatment.

\section{FUNDING AND DISCLOSURE}

The authors declare no competing interests.

\section{ACKNOWLEDGEMENTS}

This work was supported by NIH grant R01NS065926 (TJP).

\section{ADDITIONAL INFORMATION}

Supplementary Information accompanies this paper at (https://doi.org/10.1038/ s41386-019-0537-y).

Publisher's note Springer Nature remains neutral with regard to jurisdictional claims in published maps and institutional affiliations.

\section{REFERENCES}

1. Eccleston C. Chronic pain and distraction: an experimental investigation into the role of sustained and shifting attention in the processing of chronic persistent pain. Behav Res Ther. 1995;33:391-405.

2. Dick B, Eccleston C, Crombez G. Attentional functioning in fibromyalgia, rheumatoid arthritis, and musculoskeletal pain patients. Arthritis Rheum. 2002;47: 639-44.

3. Karp JF, Reynolds CF 3rd, Butters MA, Dew MA, Mazumdar S, Begley AE, et al. The relationship between pain and mental flexibility in older adult pain clinic patients. Pain Med. 2006;7:444-52.

4. Povedano M, Gascon J, Galvez R, Ruiz M, Rejas J. Cognitive function impairment in patients with neuropathic pain under standard conditions of care. J Pain Symptom Manage. 2007;33:78-89.

5. Schiltenwolf M, Akbar M, Hug A, Pfuller U, Gantz S, Neubauer E, et al. Evidence of specific cognitive deficits in patients with chronic low back pain under long-term substitution treatment of opioids. Pain Physician. 2014;17:9-20.

6. Shiers S, Pradhan G, Mwirigi J, Mejia G, Ahmad A, Kroener S, et al. Neuropathic pain creates an enduring prefrontal cortex dysfunction corrected by the type II diabetic drug metformin but not by gabapentin. J Neurosci. 2018;38:7337-50.

7. Attridge N, Pickering J, Inglis M, Keogh E, Eccleston C. People in pain make poorer decisions. Pain. 2019;160:1662-69.

8. Dick BD, Rashiq S. Disruption of attention and working memory traces in individuals with chronic pain. Anesth Analg. 2007;104:1223-9.

9. Galvez R, Marsal C, Vidal J, Ruiz M, Rejas J. Cross-sectional evaluation of patient functioning and health-related quality of life in patients with neuropathic pain under standard care conditions. Eur J Pain. 2007;11:244-55.

10. Melemedjian OK, Asiedu MN, Tillu DV, Sanoja R, Yan J, Lark A, et al. Targeting adenosine monophosphate-activated protein kinase (AMPK) in preclinical models reveals a potential mechanism for the treatment of neuropathic pain. Mol Pain. 2011;7:70

11. Moy JK, Khoutorsky A, Asiedu MN, Black BJ, Kuhn JL, Barragan-Iglesias P, et al. The MNK-elF4E signaling axis contributes to injury-induced nociceptive plasticity and the development of chronic pain. J Neurosci. 2017;37:7481-99.

12. Moy JK, Kuhn JL, Szabo-Pardi TA, Pradhan G, Price TJ. elF4E phosphorylation regulates ongoing pain, independently of inflammation, and hyperalgesic priming in the mouse CFA model. Neurobiol Pain. 2018;4:45-50.

13. Inyang KE, Szabo-Pardi T, Wentworth E, McDougal TA, Dussor G, Burton MD, et al. The antidiabetic drug metformin prevents and reverses neuropathic pain and spinal cord microglial activation in male but not female mice. Pharmacol Res. 2019;139:1-16

14. Megat S, Ray PR, Moy JK, Lou TF, Barragan-lglesias P, Li Y, et al. Nociceptor translational profiling reveals the ragulator-Rag GTPase complex as a critical generator of neuropathic pain. J Neurosci. 2019;39:393-411.

15. Yang Q, Wu Z, Hadden JK, Odem MA, Zuo Y, Crook RJ, et al. Persistent pain after spinal cord injury is maintained by primary afferent activity. J Neurosci. 2014;34:10765-9.

16. Cowie AM, Moehring F, O'Hara C, Stucky CL. Optogenetic inhibition of CGRPalpha sensory neurons reveals their distinct roles in neuropathic and incisional pain. J Neurosci. 2018;38:5807-25.

17. North RY, Li Y, Ray P, Rhines LD, Tatsui CE, Rao G, et al. Electrophysiological and transcriptomic correlates of neuropathic pain in human dorsal root ganglion neurons. Brain. 2019;142:1215-26.

18. Khoutorsky A, Price TJ. Translational control mechanisms in persistent pain. Trends Neurosci. 2018;41:100-14.

19. Uttam S, Wong C, Price TJ, Khoutorsky A. elF4E-dependent translational control: a central mechanism for regulation of pain plasticity. Front Genet. 2018; 9:470. 
20. Furic L, Rong L, Larsson O, Koumakpayi IH, Yoshida K, Brueschke A, et al. elF4E phosphorylation promotes tumorigenesis and is associated with prostate cancer progression. Proc Natl Acad Sci USA. 2010;107:14134-9.

21. Gkogkas CG, Khoutorsky A, Cao R, Jafarnejad SM, Prager-Khoutorsky M, Giannakas $\mathrm{N}$, et al. Pharmacogenetic inhibition of elF4E-dependent Mmp9 mRNA translation reverses fragile $X$ syndrome-like phenotypes. Cell Rep. 2014;9:1742-55.

22. Amorim IS, Kedia S, Kouloulia S, Simbriger K, Gantois I, Jafarnejad SM, et al. Loss of elF4E phosphorylation engenders depression-like behaviors via selective mRNA translation. J Neurosci. 2018;38:2118-33.

23. Moy JK, Khoutorsky A, Asiedu MN, Dussor G, Price TJ. elF4E phosphorylation influences Bdnf mRNA translation in mouse dorsal root ganglion neurons. Front Cell Neurosci. 2018;12:29.

24. Ueda $T$, Watanabe-Fukunaga $R$, Fukuyama $H$, Nagata $S$, Fukunaga R. Mnk2 and Mnk1 are essential for constitutive and inducible phosphorylation of eukaryotic initiation factor $4 \mathrm{E}$ but not for cell growth or development. Mol Cell Biol. 2004;24:6539-49.

25. Jackson-Laboratory. Body Weight Information for C57BL/6J. 2019.

26. Decosterd I, Woolf CJ. Spared nerve injury: an animal model of persistent peripheral neuropathic pain. Pain. 2000;87:149-58.

27. Chaplan SR, Bach FW, Pogrel JW, Chung JM, Yaksh TL. Quantitative assessment of tactile allodynia in the rat paw. J Neurosci Methods. 1994;53:55-63.

28. Angoa-Perez M, Kane MJ, Briggs DI, Francescutti DM, Kuhn DM. Marble burying and nestlet shredding as tests of repetitive, compulsive-like behaviors in mice. J Vis Exp. 2013;82:50978.

29. Black BJ, Atmaramani R, Kumaraju R, Plagens S, Romero-Ortega M, Dussor G, et al. Adult mouse sensory neurons on microelectrode arrays exhibit increased spontaneous and stimulus-evoked activity in the presence of interleukin-6. J Neurophysiol. 2018;120:1374-85.

30. King T, Vera-Portocarrero L, Gutierrez T, Vanderah TW, Dussor G, Lai J, et al. Unmasking the tonic-aversive state in neuropathic pain. Nat Neurosci. 2009;12: 1364-6.

31. Reich SH, Sprengeler PA, Chiang GG, Appleman JR, Chen J, Clarine J, et al Structure-based design of pyridone-aminal eFT508 targeting dysregulated translation by selective mitogen-activated protein kinase interacting kinases 1 and 2 (MNK1/2) inhibition. J Med Chem. 2018;61:3516-40.

32. Grubb MS, Burrone J. Activity-dependent relocation of the axon initial segment fine-tunes neuronal excitability. Nature. 2010;465:1070-4

33. Kuba $\mathrm{H}$, Oichi $\mathrm{Y}$, Ohmori $\mathrm{H}$. Presynaptic activity regulates $\mathrm{Na}(+)$ channel distribution at the axon initial segment. Nature. 2010;465:1075-8.

34. Grubb MS, Shu Y, Kuba H, Rasband MN, Wimmer VC, Bender KJ. Shortand long-term plasticity at the axon initial segment. J Neurosci. 2011; 31:16049-55.

35. Richards GC, Lluka LJ, Smith MT, Haslam C, Moore B, O'Callaghan J, et al. Effects of long-term opioid analgesics on cognitive performance and plasma cytokine concentrations in patients with chronic low back pain: a cross-sectional pilot study. Pain Rep. 2018;3:e669.

36. APF. (American Pain Foundation, 2006).

37. Apkarian AV, Sosa Y, Sonty S, Levy RM, Harden RN, Parrish TB, et al. Chronic back pain is associated with decreased prefrontal and thalamic gray matter density. J Neurosci. 2004;24:10410-5.

38. Schmidt-Wilcke T, Leinisch E, Straube A, Kampfe N, Draganski B, Diener HC, et al. Gray matter decrease in patients with chronic tension type headache. Neurology. 2005;65:1483-6.

39. Davis KD, Pope G, Chen J, Kwan CL, Crawley AP, Diamant NE. Cortical thinning in IBS: implications for homeostatic, attention, and pain processing. Neurology. 2008;70:153-4.

40. Kuchinad A, Schweinhardt P, Seminowicz DA, Wood PB, Chizh BA, Bushnell MC Accelerated brain gray matter loss in fibromyalgia patients: premature aging of the brain? J Neurosci. 2007;27:4004-7.
41. Metz AE, Yau HJ, Centeno MV, Apkarian AV, Martina M. Morphological and functional reorganization of rat medial prefrontal cortex in neuropathic pain. Proc Natl Acad Sci USA. 2009;106:2423-8.

42. Seminowicz DA, Laferriere AL, Millecamps M, Yu JS, Coderre TJ, Bushnell MC. MR structural brain changes associated with sensory and emotional function in a rat model of long-term neuropathic pain. Neuroimage. 2009;47:1007-14.

43. Ji G, Sun H, Fu Y, Li Z, Pais-Vieira M, Galhardo V, et al. Cognitive impairment in pain through amygdala-driven prefrontal cortical deactivation. J Neurosci. 2010;30:5451-64.

44. Zhang Z, Gadotti VM, Chen L, Souza IA, Stemkowski PL, Zamponi GW. Role of prelimbic GABAergic circuits in sensory and emotional aspects of neuropathic pain. Cell Rep. 2015;12:752-9.

45. Kelly $\mathrm{CJ}$, Huang $M$, Meltzer $H$, Martina $M$. Reduced glutamatergic currents and dendritic branching of layer 5 pyramidal cells contribute to medial prefrontal cortex deactivation in a rat model of neuropathic pain. Front Cell Neurosci. 2016;10:133.

46. Cheriyan J, Sheets PL. Altered excitability and local connectivity of mpfc-pag neurons in a mouse model of neuropathic pain. J Neurosci. 2018;38:4829-39.

47. Gauriau C, Bernard JF. Pain pathways and parabrachial circuits in the rat. Exp Physiol. 2002;87:251-8.

48. Goncalves L, Dickenson AH. Asymmetric time-dependent activation of right central amygdala neurones in rats with peripheral neuropathy and pregabalin modulation. Eur J Neurosci. 2012;36:3204-13.

49. Neugebauer V, Li W, Bird GC, Han JS. The amygdala and persistent pain. Neuroscientist. 2004;10:221-34.

50. Amorim IS, Lach G, Gkogkas CG. The role of the eukaryotic translation initiation factor 4E (elF4E) in neuropsychiatric disorders. Front Genet. 2018;9:561.

51. Aguilar-Valles A, Haji N, De Gregorio D, Matta-Camacho E, Eslamizade MJ, Popic J, et al. Translational control of depression-like behavior via phosphorylation of eukaryotic translation initiation factor 4E. Nat Commun. 2018;9:2459.

52. Abrahamsen B, Zhao J, Asante CO, Cendan CM, Marsh S, Martinez-Barbera JP, et al. The cell and molecular basis of mechanical, cold, and inflammatory pain. Science. 2008;321:702-5.

53. Knowlton WM, Palkar R, Lippoldt EK, McCoy DD, Baluch F, Chen J, et al. A sensorylabeled line for cold: TRPM8-expressing sensory neurons define the cellular basis for cold, cold pain, and cooling-mediated analgesia. J Neurosci. 2013;33:2837-48.

54. Price TJ, Prescott SA. Inhibitory regulation of the pain gate and how its failure causes pathological pain. Pain. 2015;156:789-92.

55. Leterrier C. The axon initial segment: an updated viewpoint. J Neurosci. 2018;38:2135-45

56. Kuba H, Yamada R, Ishiguro G, Adachi R. Redistribution of Kv1 and Kv7 enhances neuronal excitability during structural axon initial segment plasticity. Nat Commun. 2015;6:8815.

57. Mitric M, Seewald A, Moschetti G, Sacerdote P, Ferraguti F, Kummer KK, et al. Layer- and subregion-specific electrophysiological and morphological changes of the medial prefrontal cortex in a mouse model of neuropathic pain. Sci Rep. 2019;9:9479.

58. Seminowicz DA, Shpaner M, Keaser ML, Krauthamer GM, Mantegna J, Dumas JA, et al. Cognitive-behavioral therapy increases prefrontal cortex gray matter in patients with chronic pain. J Pain. 2013;14:1573-84.

59. Rodriguez-Raecke R, Niemeier A, Ihle K, Ruether W, May A. Brain gray matter decrease in chronic pain is the consequence and not the cause of pain. J Neurosci. 2009;29:13746-50.

60. Rodriguez-Raecke R, Niemeier A, Ihle K, Ruether W, May A. Structural brain changes in chronic pain reflect probably neither damage nor atrophy. PLoS ONE. 2013;8:e54475.

61. Seminowicz DA, Wideman TH, Naso L, Hatami-Khoroushahi Z, Fallatah S, Ware $M A$, et al. Effective treatment of chronic low back pain in humans reverses abnormal brain anatomy and function. J Neurosci. 2011;31:7540-50. 